\title{
Variações na coloração de iraras (Eira barbara Linnaeus, 1758 - Carnivora, Mustelidae) da Reserva Biológica Estadual do Sassafrás, Santa Catarina, sul do Brasil
}

\author{
Fernando Rodrigo Tortato ${ }^{1,2}$ \& Sérgio Luiz Althoff ${ }^{1}$ \\ Biota Neotropica $v 7$ (n3) \\ http://www.biotaneotropica.org.br/v7n3/pt/abstract?short-communication+bn02007032007 \\ Recebido em 12/10/06 \\ Versão Reformulada recebida em 05/09/07 \\ Publicado em 23/09/07 \\ ${ }^{1}$ Laboratório de Biologia Animal, Departamento de Ciências Naturais - DCN, \\ Centro de Ciências Exatas e Naturais - CCEN, Campus I, \\ Fundação Universidade Regional de Blumenau - FURB, Rua Antônio da Veiga, 140, \\ Bairro Victor Konder, CEP 89012-900, Blumenau, Santa Catarina, Brasil \\ ${ }^{2}$ Autor para correspondência: Fernando Rodrigo Tortato, e-mail:ftortato@hotmail.com
}

\begin{abstract}
Tortato, F.R \& Althoff, S.L. Variation in coat color of tayras (Eira barbara Linnaeus, 1758 - Carnivora, Mustelidae) in the State Biological Reserve of Sassafrás, Santa Catarina, South Brazil. Biota Neotrop. Sep/Dez 2007 vol. 7, no. 3 http://www.biotaneotropica.org.br/v7n3/pt/abstract?shortcommunication+bn02007032007. ISSN 1676-0603.

Tayras present color variations along their range of distribution. The objective of this study is to contribute to the understanding of these variations. In the State Biological Reserve of Sassafrás, Santa Catarina, Brazil, 21 photographic records of tayras were obtained, comprising different coat colors. Two coat color classes were observed among photographed individuals: dark body with gray head and neck, and body, head and neck homogeneously white-yellowish. The frequency of records did not varied significantly between the two color classes, indicating that the white-yellowish coloration is common for this local population.

Keywords: color variation, tayra, coat color, Atlantic Rain Forest.

Resumo

Tortato, F.R \& Althoff, S.L. Variações na coloração de iraras (Eira barbara Linnaeus, 1758 - Carnivora, Mustelidae) da Reserva Biológica Estadual do Sassafrás, Santa Catarina, sul do Brasil. Biota Neotrop. Sep/Dez 2007 vol. 7, no. 3 http://www.biotaneotropica.org.br/v7n3/pt/abstract?shortcommunication+bn02007032007. ISSN 1676-0603.

Iraras apresentam variações de coloração por toda sua área de distribuição, sendo o objetivo deste estudo contribuir para o conhecimento destas variações. Na Reserva Biológica Estadual do Sassafrás, Santa Catarina, Brasil, foram obtidos 21 registros fotográficos de iraras, abrangendo diferentes colorações de pelagem. Duas classes de coloração foram observadas entre os indivíduos fotografados: corpo escuro com cabeça e pescoço cinzas e corpo, cabeça e pescoço branco-amarelados. A freqüência dos registros não variou significativamente entre as duas classes de coloração, indicando que a coloração de pelagem branco-amarelada é comum para esta população local.
\end{abstract}

Palavras-chave: variação de coloração, irara, cor da pelagem, Mata Atlântica. 


\section{Introdução}

A coloração nos mamíferos está comumente relacionada à camuflagem, comunicação intra e interespecífica e funções físicofisiológicas (Ortolani 1999, Caro 2005). Variações fenotípicas ao longo da área de distribuição podem estar relacionadas a variações climáticas sazonais, faixa etária, sexo, peso, entre outros (Ortolani 1999, Stoner et al. 2003, Bordignon \& França 2004, Caro 2005).

A irara, Eira barbara, ocorre desde o México até o norte da Argentina, possui hábito noturno ou crepuscular, solitário e geralmente é encontrada em áreas florestadas (Cabrera \& Yepes 1960, Emmons 1997, Eisenberg \& Redford 1999, Presley 2000). O corpo é esguio e musculoso, a cauda longa e as orelhas pequenas e redondas (Emmons 1997, Presley 2000). A coloração pode variar ao longo de sua área de distribuição. Ao longo de toda distribuição, predominam cores escuras na pelagem (Cabrera \& Yepes 1960, Emmons 1997, Eisenberg \& Redford 1999, Presley 2000). Porém, no Peru, a cabeça é mais escura que o restante do corpo, enquanto que na costa do Equador (Cabrera \& Yepes 1960, Emmons 1997, Eisenberg \& Redford 1999, Presley 2000) e no sul do Brasil (Silva 1994, Cimardi 1996) os exemplares comumente apresentam a cabeça clara e o restante do corpo escuro.

Estas variações de coloração regionais levaram diversos autores a descrever sete subespécies reconhecidas por Cabrera (1958) e Hall (1981), e sumarizadas por Presley (2000). A subespécie que ocorre nas regiões centro-oeste, sudeste e sul do Brasil, Argentina, Paraguai, Bolívia e Peru é Eira barbara barbara, cujo corpo é marrom, apresentando uma gradação de marrom para cinza do corpo para cabeça com uma mancha amarelada na parte inferior do pescoço (Presley 2000).

Indivíduos com padrões de pelagem apresentando coloração branco-amarelada já foram descritos por alguns autores (Cabrera \& Yepes 1960, Emmons 1997, Presley 2000). Presley (2000) menciona que nas Guianas ocorre tanto a forma de pelagem amarela como a escura. Tarifa et al. (2001) relatam à presença de indivíduos amarelo-claros no Parque Nacional Madidi, Bolívia. No Brasil, foram publicados somente dois estudos mencionando a ocorrência dessa pelagem em iraras, sendo um referente a um indivíduo de pelagem branco-amarelada registrado com armadilha fotográfica na região do Rio Jauaperí, no estado do Amazonas (Trolle 2003), e outro na Fazenda Monte Alegre, no estado do Paraná, onde foram visualizados esporadicamente indivíduos de coloração quase branca, mas que não foram considerados albinos (Reis et al. 2005). Neste trabalho, apresentamos novo registro de iraras de pelagem clara para o estado de Santa Catarina, e através do uso de armadilhas fotográficas, comparamos a frequiência de ocorrência de diferentes classes de pelagem das iraras na Reserva Biológica Estadual do Sassafrás, Santa Catarina, sul do Brasil.

\section{Material e métodos}

Entre setembro de 2004 e novembro de 2006, realizou-se um inventário de mamíferos na Reserva Biológica Estadual do Sassafrás (RBES), situada ente as coordenadas $26^{\circ} 42^{\prime \prime} \mathrm{S}$ e $49^{\circ} 40^{\prime} \mathrm{W}$, através do uso de armadilhas fotográficas. A RBES está localizada na Bacia do Rio Itajaí-Açu, leste do estado de Santa Catarina e possui uma área de 3.862 hectares, coberta por vegetação de transição entre a Floresta Ombrófila Densa e a Floresta Ombrófila Mista.

Os registros de iraras foram obtidos com armadilhas fotográficas (Tigrinus ${ }^{\circledR}$ ) em seis de um total de 17 estações de captura e esforço de 660 armadilhas-dia. Durante a maior parte do trabalho de campo foi utilizada somente uma armadilha fotográfica, e no último ano duas armadilhas. Estas eram instaladas em trilhas e estradas onde eram encontrados indícios da presença de mamíferos de maior porte a, no mínimo, $300 \mathrm{~m}$ uma da outra. As armadilhas fotográficas estavam programadas com um intervalo de um minuto entre uma fotografia e outra. Em duas ocasiões, em que ocorreram registros de iraras foi utilizada maçã como isca. Foram considerados registros fotográficos independentes do mesmo padrão de coloração de pelagem, aqueles que apresentaram intervalos iguais ou superiores a 24 horas.

Para analisar a variação de coloração das iraras, duas classes foram consideradas, uma com corpo escuro e cabeça e pescoço cinzas (Figura 1) e outra com corpo, cabeça e pescoço brancoamarelados (Figura 2). Entre os registros de indivíduos escuros, avaliou-se a presença da mancha amarelada na parte inferior do pescoço, característica de E. barbara barbara. O teste qui-quadrado $\left(\chi^{2}\right)$ com a correção de Yates para pequenas amostras (Zar 1999) foi utilizado para verificar se havia variação significativa na freqüência de registro entre as classes de coloração. Informações obtidas de entrevistas com moradores do entorno da RBES também foram consideradas.

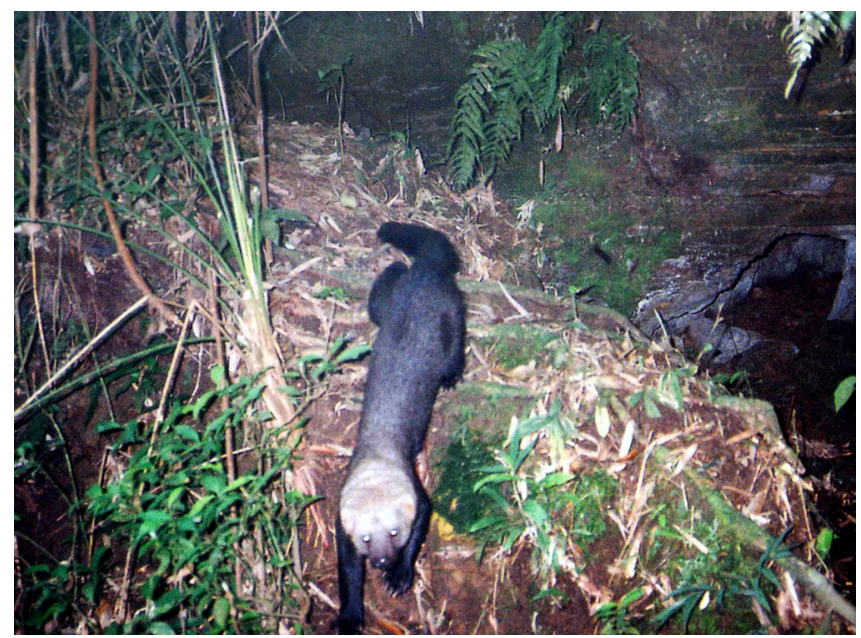

Figura 1. Irara (Eira barbara) com corpo escuro e cabeça e pescoço cinzas, registrada na Reserva Biológica Estadual do Sassafrás, Santa Catarina, sul do Brasil.

Figure 1. Tayra (Eira barbara) with dark body and gray head and neck, recorded in the State Biological Reserve of Sassafrás, Santa Catarina, south Brazil.

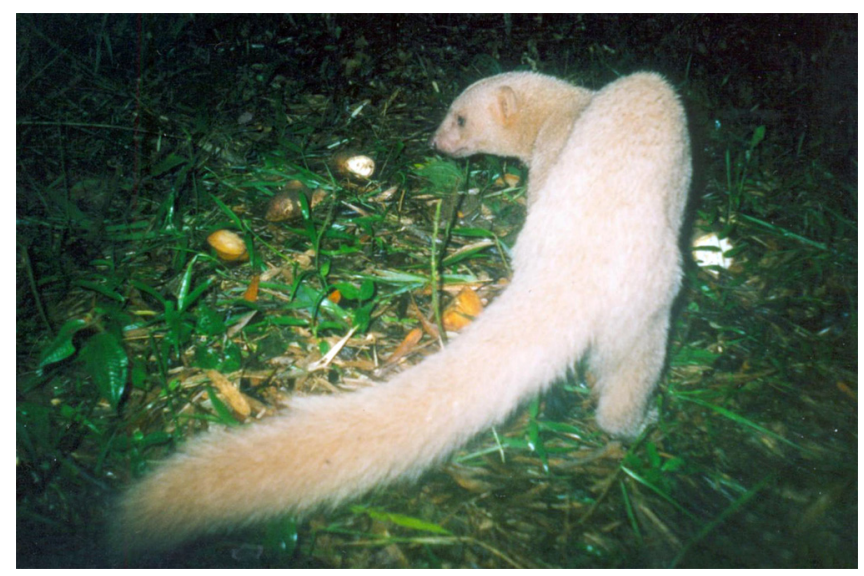

Figura 2. Irara (Eira barbara) com coloração branco-amarelada, registrada na Reserva Biológica Estadual do Sassafrás, Santa Catarina, sul do Brasil.

Figure 2. Tayra (Eira barbara) with white-yellowish coloration, recorded in the State Biological Reserve of Sassafrás, Santa Catarina, south Brazil. 


\section{Resultados e Discussão}

Foram obtidos 21 registros de iraras, sendo seis de indivíduos de coloração branco-amarelada e 15 de indivíduos escuros. Foi possível analisar a presença ou ausência de manchas amareladas sob o pescoço de seis dos 15 registros de coloração escura, sendo que destes apenas dois $(33,33 \%)$ apresentaram manchas.

Não se verificou diferença significativa entre as frequiências de registro das classes de coloração analisadas $\left(\chi^{2}=3,04 ; \mathrm{p}>0,05\right)$. A proporção calculada foi de 2,67:1. Obtivemos, ainda, três relatos de iraras amareladas, avistadas em ocasiões e lugares distintos na Reserva.

A coloração de pelagem amarelada observada na Reserva Biológica Estadual do Sassafrás não pode ser devida a albinismo. O albinismo é caracterizado pela ausência total de melanina, causado por uma anomalia recessiva, tendo como particularidade à cor rosada da pele, olhos vermelhos e, nos mamíferos, uma pelagem branca (Veiga \& Pardo 1990, Sazima \& Di Bernardo 1991, Veiga 1994, Cademartori \& Pacheco 1999, Rodrigues et al. 1999). Todos os indivíduos fotografados apresentaram as extremidades das patas e da cauda, o focinho e os olhos pigmentados.

Apesar do número de registros de iraras de coloração brancoamarelada ter sido menor do que o de iraras de coloração escura, a proporção do número de registros entre as classes de pelagem não diferiu significativamente de 1:1, o que, somado aos relatos de moradores locais, indica que a coloração branco-amarelada é relativamente comum na área de estudo. De fato, vários autores (Cabrera \& Yepes 1960, Silva 1994, Cimardi 1996, Emmons 1997, Eisenberg \& Redford 1999, Presley 2000) reportam variações de coloração de pelagem nas iraras. Desta forma, variações nas colorações e presença ou ausência de manchas sob o pescoço são características frágeis para serem utilizadas em estudos taxonômicos.

\section{Agradecimentos}

Os autores agradecem a Fundação do Meio Ambiente de Santa Catarina (FATMA), Modo Battistella Reflorestamento S/A MOBASA e Tigrinus Equipamentos para Pesquisa Ltda. pelo apoio logístico e a Walfrido M. Tomas, Marcos A. Tortato, Rudi R. Laps, Geraldo Moretto, Alexandre Uhlmann e Adrian E. Rupp pelas sugestões e correções deste trabalho.

\section{Referências Bibliográficas}

BORDIGNON, M.O. \& FRANÇA, A.O 2004. Variações na coloração da pelagem do morcego-pescador Noctilio leporinus (L., 1758) (Mammalia, Chiroptera). Rev Bras de Zooc 6(2):181-189.
CABRERA, A. 1958. Catálogo de los mamíferos de América del Sur. v. 1. Rev Mus Argent Ci Nat. "Bernardino Rivadavia", Ci. Zool. 4(1):1-307.

CABRERA, A. \& YEPES, J. 1960. Mamíferos Sud Americanos. v.1. 2 ed. Ediar S.A. Editores.

CADEMARTORI, C.V. \& PACHECO, S.M. 1999. Registro de albinismo em Delomys dorsalis (Hensel, 1872) - Cricetidae, Sigmodontinae. Biociências 7(1):195-197.

CARO, T. 2005. The adaptive significance of coloration in mammals. Bioscience 55(2):125-136.

CIMARDI, A.V. 1996. Mamíferos de Santa Catarina. Fundação de Amparo à Tecnologia e Meio Ambiente, Florianópolis.

EISENBERG, J.F. \& REDFORD, K.H. 1999. Mammals of the Neotropics. v.3. The Central Neotropics: Ecuador, Peru, Bolivia, Brazil. The University of Chicago Press, Chicago.

EMMONS, L.H. 1997. Neotropical rainforest mammals. A field guide. 2 ed. The University of Chicago Press, Chicago

HALL, E. R. 1981. The mammals of North America. $2^{\text {nd }}$ ed. John Wiley \& Sons, New York.

ORTOLANI, A. 1999. Spots, stripes, tail tips and dark eyes: Predicting the function of carnivore colour patterns in carnivores using the comparative method. Biol J Linn Soc 67:433-476.

PRESLEY, S. J. 2000. Eira barbara. Mammalian Species 636:1-6.

REIS, N.R., PERACCHI, A.L., FANDIÑO-MARINO, H. \& ROCHA, V.J. 2005. Mamíferos da Fazenda Monte Alegre - Paraná. Eduel, Londrina.

RODRIGUES, F.H.G., SILVEIRA, L., JÁCOMO, A.T. \& MONTEIRO-FILHO E.L.A. 1999. Um albino parcial de veado campeiro (Ozotocerus bezoarticus, Linnaeus) no Parque Nacional das Emas, Goiás. Rev Bras Zool, 16(4):1229-1232.

SAZIMA, I. \& DI-BERNARDO, M. 1991. Albinismo em serpentes neotropicais. Mem I Butantan 53(2):167-173.

SILVA, F. 1994. Os mamíferos do Rio Grande do Sul. Fundação Zoobotânica do Rio Grande do Sul, Porto Alegre.

STONER, C.J., BININDA-EMONDS, O.R.P. \& CARO, T. 2003. The adaptive significance of colouration in lagomorphs. Biol J Linn Soc 79:309-328.

TARIFA, T., ALIAGA E., RÍOS, B. \& HAGAMAN, D. 2001. Mamíferos del Parque Nacional Madidi. Hisbol. La Paz.

TROLLE, M. 2003. Mammal survey in the Rio Jauaperí region, Rio Negro Basin, the Amazon, Brazil. Mammalia 67(1):75-83.

VEIGA, L. A. \& PARDO, E. 1990. Ocorrência de um caso de albinismo em Sabiá Laranjeira. Arq Bio Tec 33:329-333.

VEIGA, L. A. 1994. Um caso de albinismo em Tayassu tajacu Linnaeus (Artiodactyla, Tayassuidae) na Serra do Mar, São José dos Pinhais, Paraná. Rev Bras Zool 11(2):341-343.

ZAR, J. H. 1999. Biostatistical Analysis. 4. ed. Prentice-Hall, Englewood Cliffs, New Jersey, USA. 
